\title{
Epigenetic DNA hypermethylation: Clinical applications in endometrial cancer (Review)
}

\author{
YURIKO MURAKI, KOUJI BANNO, MEGUMI YANOKURA, YUSUKE KOBAYASHI, MAKIKO KAWAGUCHI, \\ HIROYUKI NOMURA, AKIRA HIRASAWA, NOBUYUKI SUSUMU and DAISUKE AOKI
}

Department of Obstetrics and Gynecology, Keio University School of Medicine, Tokyo 160-8582, Japan

Received May 6, 2009; Accepted June 22, 2009

DOI: 10.3892/or_00000523

\begin{abstract}
Improvements in epigenetics have resulted in identification of a number of genes with aberrant hypermethylation associated with systematic occurrence of cancer. It is now evident that aberrant hypermethylation inactivates cancer-related genes including those associated with cell cycle control, apoptosis, and DNA repair. An epigenetic analysis of DNA hypermethylation in type I endometrial cancer has led to a proposed mechanism for endometrial carcinogenesis. Reduced DNA mismatch repair due to loss of $h M L H 1$ expression is thought to have a major role in carcinogenesis and these findings open up approaches to prevention, diagnosis, risk assessment, and treatment of type I endometrial cancer. Aberrant DNA hypermethylation can be detected with high sensitivity for identification of cancer cells in sputum, blood and other biopsy materials, including in endometrial cancer specimens. There have been many attempts to use methylation inhibitors as anticancer agents, and epigenetic abnormalities may be useful as biomarkers of anticancer drug sensitivity and to identify biological characteristics of tumor cells for determination of treatment options based on hypermethylation. For example, aberrant hypermethylation of the CHFR gene is correlated with cellular sensitivity to microtubule inhibitors, and this may be useful in treatment of type I endometrial cancer. An ultimate objective of epigenetics is to identify the type of hereditary methylation responsible for cancer, with the goal of improved diagnosis and treatment based on control of methylation.
\end{abstract}

\section{Contents}

1. Introduction

2. Epigenetic DNA hypermethylation in cancer cells

Correspondence to: Dr Kouji Banno, Department of Obstetrics and Gynecology, Keio University School of Medicine, Shinanomachi 35, Shinjuku-ku, Tokyo 160-8582, Japan

E-mail: kbanno@sc.itc.keio.ac.jp

Key words: endometrial cancer, DNA hypermethylation, epigenetics, $h M L H 1, C H F R$
3. Epigenetic DNA hypermethylation in endometrial cancer

4. Application of aberrant DNA hypermethylation to diagnostics

5. Application of aberrant DNA hypermethylation to treatment

6. Future directions and conclusion

\section{Introduction}

Epigenetics refers to the information stored after somatic cell division that is not contained within the DNA base sequence. Recent findings have shown that epigenetic changes, selective abnormalities in gene function that are not due to DNA base sequence abnormalities, play a significant role in carcinogenesis in various organs. In particular, the relationship between cancer and aberrant hypermethylation of specific genome regions has attracted attention. A completely new model for the mechanism of carcinogenesis has been proposed in which hypermethylation of unmethylated $\mathrm{CpG}$ islands in the promoter regions of cancer-related genes in normal cells silences these genes and leads to the cell becoming cancerous (Fig. 1). Both genetic and epigenetic changes are intricately involved in the process through which cells become cancerous, and hypermethylation of cancer-related genes such as p16, $A P C$, and $h M L H 1$ has been associated with several types of cancer $(1,2)$. The main difference between epigenetic abnormalities and genetic abnormalities such as gene mutations is that epigenetic changes are reversible and do not involve changes in base sequence, which suggests that gene reexpression is possible and that epigenetic data may lead to important molecular targets for treatment. Attempts have begun to detect aberrant DNA methylation of cancer cells present in minute quantities in biological samples and to apply the results to cancer diagnosis, prediction of the risk of carcinogenesis, and definition of the properties of a particular cancer.

In Japan, the number of women with endometrial cancer and the prevalence and mortality rate of this cancer continue to increase due to Westernization of lifestyles and environmental changes. Endometrial cancer currently accounts for approximately $40 \%$ of all cancers of the uterus and an increase in the total number of patients and the number of young women with this condition has been forecasted. Elucidation of the pathogenesis and establishment of effective 
Normal Gene

Unmethylated CpG site

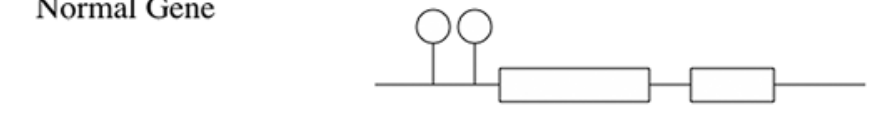

Genetic Abnormality

A. Mutation

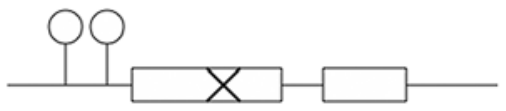

B. Deletion

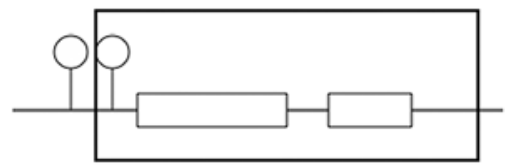

Epigenetic Abnormality

C. Hypermethylation in Promoter Region

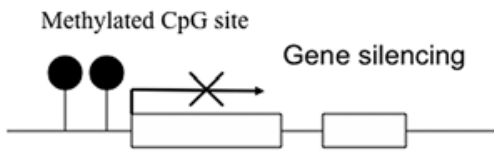

Figure 1. Mechanism of inactivation of cancer-related genes

treatment for endometrial cancer are significant challenges in gynecological oncology, but many aspects of the carcinogenic mechanism are still poorly understood. The conventional explanation of the mechanism involving genetic changes, mutations of cancer-related genes, is inadequate and epigenetic changes in endometrial cancer are now being examined. In particular, aberrant DNA methylation is thought to play a key role in endometrial carcinogenesis. Breakdown of the DNA mismatch repair mechanism plays a particularly important role in the development of type I endometrial cancer, and inhibition of $h M L H 1$ expression due to DNA methylation may contribute significantly to this mechanism. Therefore, an understanding of the epigenetics of DNA methylation may shed light on the mechanism of carcinogenesis and improve diagnosis, risk evaluation, treatment, and prevention of endometrial cancer.

\section{Epigenetic DNA hypermethylation in cancer cells}

Mechanisms involved in epigenetic regulation of gene expression involve DNA methylation, histone modification, and polycomb-group proteins (3). DNA methylation patterns are faithfully stored after cell division and DNA methylation is one of the most common and best studied epigenetic modifications in mammals. Genomic DNA methylation in vertebrates occurs at the cytosine in $\mathrm{CpG}$ sites; that is, where a cytosine is directly followed by a guanine in the DNA sequence. Transfer of the methyl group from S-adenosyl-L-methionine is catalyzed by DNA methyltransferase enzymes in two distinct processes referred to as maintenance methylation and de novo methylation. Maintenance methylation takes place after DNA replication, which produces hemimethylated DNA in which only one of the strands is methylated. $\mathrm{CpG}$ sites on the daughter strand are then methylated in exactly the same way as the parent strand. In maintenance methylation, the methylation pattern of the parent strand is copied onto the daughter strand produced by DNA replication during cell division, thus allowing maintenance of the pattern. De novo methylation involves methylation of a completely unmethylated $\mathrm{CpG}$, allowing new methylation in the course of generation or differentiation of cells, aging, or neoplastic transformation. The known DNA methyltransferases Dnmt1, Dnmt2, Dnmt3a, Dnmt3b and Dnmt3L are classified according to whether they catalyze maintenance or de novo methylation. DNA methylation in a region with a dense concentration of $\mathrm{CpG}$ sites ( $\mathrm{CpG}$ islands) upstream from the transcription initiation site has a critical effect on gene expression (4). It has also been shown that a region of unmethylated DNA tends not to form nucleosomes, allowing transcription to occur, whereas methylated DNA induces nucleosome formation that renders transcription impossible $(5,6)$.

Tumor suppressor genes such as $C D K N 2 A, C D H 1$ (E-cadherin), and $h M L H 1$ are silenced as a result of aberrant DNA methylation of $\mathrm{CpG}$ islands in their promoter regions. Aberrant DNA methylation may play a significant role in carcinogenesis, as for gene mutations (7). In colorectal cancers, $\mathrm{CpG}$ island methylation is found either genomewide or in only a few genes, and Toyoda et al proposed that these two patterns should be clearly discriminated based on the carcinogenic mechanism (8). Cancers with widespread methylation covering the whole genome have a damaged methylation control mechanism and are classified as $\mathrm{CpG}$ island methylator phenotype (CIMP) cancers, in which methylation of genes is thought to occur continuously. The mechanism underlying CIMP cancers is not understood, but it is hypothesized that low-frequency promoter $\mathrm{CpG}$ methylation first occurs at a few $\mathrm{CpG}$ sites and then gradually extends over a wide area as a result of DNA replication during cell division. It is important to note that methylation continues, despite the low frequency and small proportion of CpG sites that are methylated. Even more importantly, for methylation to extend over a wider area, the gene must have low transcription activity, which suggests that a lack of gene transcription is important for methylation of promoter $\mathrm{CpG}$ islands. However, $\mathrm{CpG}$ island methylation does not always lead to gene silencing. DNA hypermethylation of tumor suppressor genes can cause carcinogenesis (driver methylation), but it is now thought that there are a large number of genes for which methylation is a result of carcinogenesis, rather than the cause of an original low transcript level (passenger methylation).

\section{Epigenetic DNA hypermethylation in endometrial cancer}

Endometrial cancer is classified into types I and II according to clinicopathological characteristics. Type I endometrial cancer mainly occurs in pre- or perimenopausal women; is estrogen dependent and positive for both estrogen and progesterone receptors; and develops from endometrial hyperplasia. Pathologically, type I endometrial cancer is a welldifferentiated endometrioid adenocarcinoma with a low incidence of lymph node metastasis and myometrial invasion. The prognosis is usually comparatively favorable. Type II endometrial cancer mainly occurs in postmenopausal women; is estrogen independent; and is thought to develop from a normal endometrium directly or from unspecified precancerous lesions, but not from endometrial hyperplasia. Histologically, it is an unusual type with poorly differentiated 


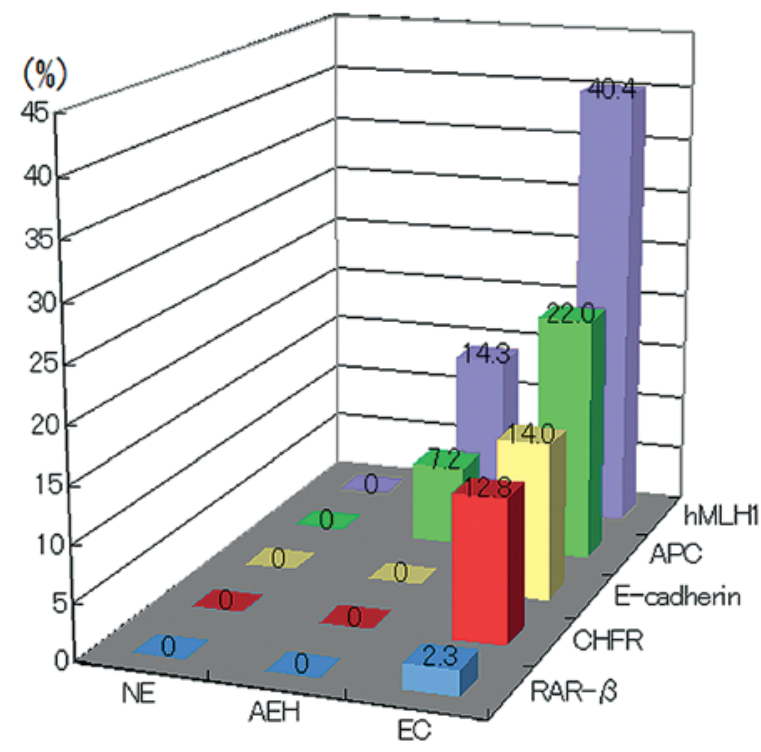

NE: Normal endometrium

AEH: Atypical endometrial hyperplasia

EC: Endometrial cancer

Figure 2. Frequency of aberrant hypermethylation of genes in oncogenesis in the endometrium, as found by Banno et al (10).

endometrioid adenocarcinoma or serous adenocarcinoma, and the prognosis is usually poor.

Different molecular mechanisms are thought to be involved in the development of the two types of endometrial cancer. An epigenetic mechanism has been proposed for development of type I endometrial cancer based on DNA mismatch repair (MMR) deficiency, which is a typical genetic defect in this cancer. The DNA mismatch repair system corrects errors in bases that arise when genes are replicated during cell division and silencing of DNA mismatch repair genes reduces the ability to repair gene mutations. This results in an accumulation of cancer-related gene mutations, leading to carcinogenesis. The MMR gene $h M L H 1$ is a typical gene that is silenced by DNA methylation. In endometrial cancer, $h M L H 1$ silencing is found in approximately $40 \%$ of cases and is an important step in the early stages of carcinogenesis, with the loss of DNA mismatch repair function proposed to lead to mutation of genes such as PTEN.

Microsatellite instability (MSI) occurs when the mismatch repair system is damaged. Microsatellites are DNA sequences of repeating units of between 1 and 5 base pairs. Abnormalities in the mismatch repair system may cause replication errors in the repeating unit, leading to changes in length that are referred to as microsatellite instability. MSI is observed in certain types of cancer, including approximately $20-30 \%$ of cases of endometrial cancer (9). These results suggest that MMR gene abnormalities occur frequently in endometrial cancer.

In patients with endometrial cancer, Banno et al found aberrant hypermethylation of $h M L H 1, A P C, E$-cadherin, and $C H F R$ in $40.4,22.0,14.0$, and $13.3 \%$ of cases, respectively. A significant decrease in protein expression was found in patients with aberrant methylation of $h M L H 1$ $(\mathrm{P}<0.01)$ and $E$-cadherin $(\mathrm{P}<0.05)$, and aberrant methylation

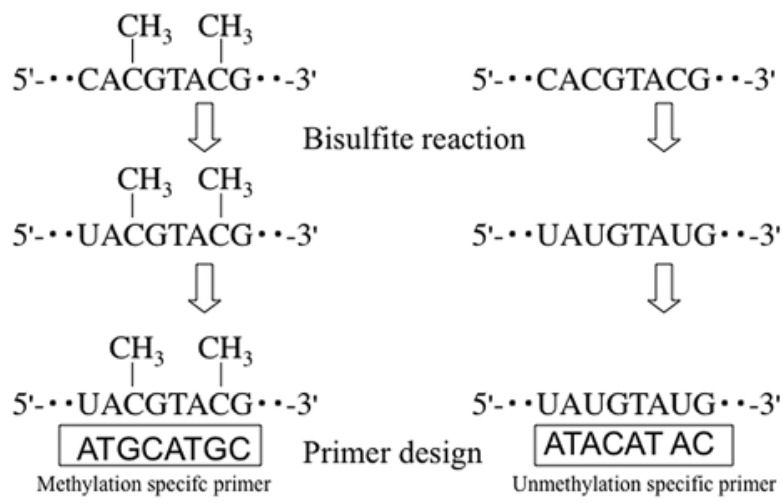

Detection with PCR

Figure 3. Principles of bisulfite treatment and methylation-specific PCR (MSP).

of $h M L H 1$ was also found in $14.3 \%$ of patients with atypical endometrial hyperplasia. However, no aberrant methylation of the four cancer-related genes was found in patients with a normal endometrium. These results indicate that aberrant methylation of specific genes associated with carcinogenesis in endometrial cancer does not occur in a normal endometrium, with aberrant methylation of the $h M L H 1$ gene being most frequent. The aberrant methylation of $h M L H 1$ in atypical endometrial hyperplasia, which is found in the first stage of endometrial cancer, supports the hypothesis that $h M L H 1$ aberrant methylation is an important event in carcinogenesis in endometrial cancer (10) (Fig. 2).

\section{Application of aberrant DNA hypermethylation to diagnostics}

Aberrant DNA methylation can be analyzed using the polymerase chain reaction (PCR), which shows a high degree of sensitivity for minute quantities of DNA in biological samples. However, it is important to ensure that the DNA hypermethylation is specific to cancer cells, since aberrant DNA hypermethylation can also occur in non-cancerous cells. Specific aberrant DNA hypermethylation may be applicable to cancer diagnosis, but use of this method for cancer screening requires detection of cancer-cell genes with aberrant hypermethylation in clinical samples that also contain normal cells. Methylation-specific PCR (MSP), which combines bisulfite sequencing and PCR, can detect aberrant hypermethylation with a high degree of sensitivity using small quantities of DNA. In bisulfite sequencing, cytosine is converted to uracil, but methylated cytosine is not converted. The PCR is thus set up with primers for sequences containing cytosine or uracil and amplification is performed to detect aberrant hypermethylation (Fig. 3). This procedure can be used with various biological samples, including sputum, plasma and urine, and has been used to detect aberrant methylation of cancer-associated genes in endometrial cell samples (10).

Aberrant DNA hypermethylation has been reported to affect several genes in endometrial cancer, in addition to $h M L H 1$. Such genes include CASP 8 (11), an apoptosisrelated gene; $T G F-\beta R I I$ (12), a TGF- $\beta$ receptor with a tumor 
suppressor effect; $p 73$ (11), a tumor suppressor gene; HOXA11 (13), which is important in uterine development; and COMT (14), which codes for the catechol-O-methyltransferase that metabolizes catechol, an estrogen metabolite that plays a role in carcinogenesis. Methylation of each of these genes results in a loss of protein expression that promotes cancer, and the degree of methylation of the genes differs significantly between normal tissues and endometrial cancer tissues (15).

The properties of cancer cells can be significantly affected by aberrant DNA hypermethylation. For example, in colorectal cancer, methylation of CHFR is observed in $40 \%$ of cases and is closely related to sensitivity to the microtubule inhibitor docetaxel (16). Other examples of similar effects include the relationship between aberrant hypermethylation of the DNA repair enzyme $M G M T$ and alkylating agent sensitivity (17), the link between simultaneous hypermethylation of CDKN2A and FHIT and recurrence of lung cancer (18), and the link between methylation of $E R-\alpha$ and prognosis during treatment with tamoxifen (19). This last example indicates that detection of aberrant hypermethylation can be used for prognostic evaluation. As discussed above, CIMP cancers have widespread promoter hypermethylation of various genes as a result of damage to the methylation control mechanism. The prognosis of neuroblastoma with CIMP has been found to be poorer than that without CIMP (20), which may be due to changes in expression of genes related to prognosis due to the damaged methylation control mechanism.

Aberrant DNA hypermethylation is sometimes seen in the elderly and in non-cancerous regions in cancer patients (21-23). In particular, it has been shown that exposure to $H$. pylori, a carcinogenic factor for gastric cancer, induces aberrant DNA hypermethylation and that the degree of aberration is correlated with carcinogenic risk. This leads to the concept of an 'epigenetic field for cancerization', with similar results reported for colorectal and breast cancer (23). Accumulation of aberrant methylation in normal tissue may trigger carcinogenesis and such information may be useful to evaluate carcinogenic risk.

\section{Application of aberrant DNA hypermethylation to treatment}

Unlike irreversible genetic changes, epigenetic patterns observed in cancer can be partly or fully reversed pharmacologically. Indeed, this is the main reason for development of anticancer drugs for epigenetic modification. Treatment of cancer using demethylating agents to restore expression of cancer suppressor genes silenced through methylation has been attempted for some time and use of methylation inhibitors to treat cancer has a long history. Development of an antileukemic agent based on this principle was performed in the 1960 s, but the drug could not be used clinically due to toxicity problems at high concentrations. More recent reports have shown antitumor effects at lower drug concentrations with a lower incidence of adverse drug reactions and concomitant use with other chemotherapeutic agents may further improve efficacy. Lubbert et al reported a response rate of $60 \%$ in patients with myelodysplastic syndrome (MDS) following administration of the methylation inhibitor 5-aza-2'-deoxy-

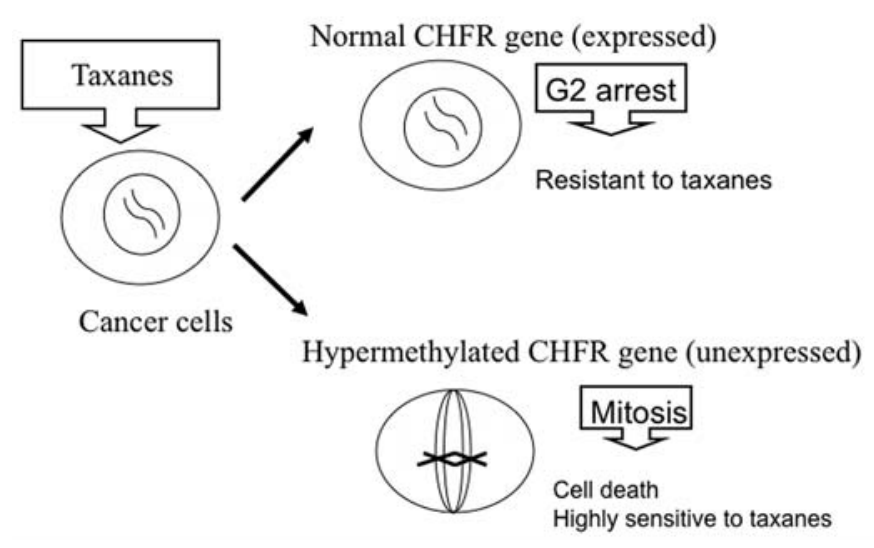

Figure 4. Hypothesis for the relationship of expression of CHFR and sensitivity of cancer cells to taxanes.

cytidine (5-aza-dC) (24), with induction of expression of p15INK4A following demethylation in patients who were responsive to 5-aza-dC. A group from MD Anderson Cancer Center reported that repeated administration of low-concentration 5-aza-dC produced a response rate of approximately $60 \%$ in patients with acute myeloid leukemia (25). However, the disadvantage of this methylation inhibitor is that it is not sequence specific, which may lead to adverse effects through demethylation of physiologically important genes and reactivation of cancer genes silenced by methylation. Therefore, development of sequence-specific demethylating agents based on binding sequence of transcription factors is a current area of research.

Epigenetic abnormalities have also been examined as markers of anti-cancer drug sensitivity. Esteller et al discovered that the DNA repair enzyme $M G M T$ gene is silenced by methylation and showed that tumor cell lines in which $M G M T$ is methylated are highly responsive to alkylating agents, with a greater anti-tumor effect of BNCU observed in patients in whom MGMT methylation was detected in the tumor compared to those with no MGMT methylation (26). Sato et al reported that aberrant hypermethylation of CHFR, a mitotic checkpoint gene, is strongly correlated with responsiveness to taxanes, which are microtubule inhibitors (27) (Fig. 4). These findings suggest that it may be possible to select a treatment based on methylation as an indicator of the biological characteristics of tumor cells. CHFR methylation may be a particularly sensitive marker in endometrial cancer and analysis of this gene may play an important role in treatment of this type of cancer. CHFR is an M-phase checkpoint gene with folk-head associated (FHA) and ring finger domains that was first identified as a yeast DMA 1 gene homolog (28). If the cell is subjected to mitotic stress in the M-phase, CHFR delays progression from the start of prophase to the later part of prophase in mitosis. CHFR is a ubiquitin ligase that includes Aurora-A and PLK1 among its substrates, and degradation of these proteins is thought to stop the cell cycle following CHFR activation by microtubular stress $(29,30)$. The FHA domain of CHFR is involved in binding of phosphorylated proteins and is important in the checkpoint function, but further studies are required to identify molecules that interact with CHFR. 
CHFR methylation is thought to occur frequently in cancer $(16,27)$ and is related to the mitotic index as follows. In normally functioning cells with no CHFR methylation, cell-cycle arrest occurs during the $\mathrm{G} 2 / \mathrm{M}$ phase following administration of docetaxel and the mitotic index is low. Conversely, in cells in which CHFR hypermethylation has reduced expression of the gene, the mitotic index is high following administration of docetaxel. These results show that the expression level of $C H F R$ is negatively correlated with the mitotic index (16). In cells in which the CHFR checkpoint does not function, translocation of cyclin B1 to the nucleus during mitotic stress cannot be prevented. Treatment of cells with $C H F R$ hypermethylation with the methylation inhibitor 5 -aza-dC restores the checkpoint function and decreases the mitotic index. CHFR methylation and microtubule inhibitor sensitivity are useful molecular markers in gastric and cervical cancer, as well as in endometrial cancer, and methylation may be a useful predictor of anti-cancer drug responsiveness (31-33).

\section{Future directions and conclusion}

Progress made in epigenetics in recent years has suggested that aberrant DNA hypermethylation plays a role in carcinogenesis in several types of cancer. Various genes are silenced as a result of aberrant hypermethylation, including cell cycle regulatory genes, apoptosis-related genes and DNA repair enzymes, and in CIMP tumors the methylation control mechanism is thought to be damaged.

Epigenetic research in endometrial cancer suggests that damage to the mismatch repair system plays a significant role in development of type I endometrial cancer and that $h M L H 1$ hypermethylation is important in this mechanism. Such research has potential for prevention, diagnosis, risk assessment, and treatment of endometrial cancer. Cancerspecific DNA methylation may be useful for diagnosis using methods such as MSP for detection of such abnormalities. Aberrant DNA hypermethylation can be detected with a high level of sensitivity and cancer cells can be detected in minute quantities of endometrial samples. Treatment with methylation inhibitors such as 5-aza-dC may also be effective, since a low concentration of this drug has an anti-tumor effect with a reduced incidence of adverse drug reactions, and concomitant use with other chemotherapy drugs may show even greater efficacy. Attempts are also being made to use epigenetic abnormalities as indicators of anti-cancer drug sensitivity, which may allow selection of the most appropriate treatment based on the biological characteristics of tumor cells. Aberrant CHFR hypermethylation is strongly findings may be applicable in treatment of endometrial cancer. The main objective of epigenetics in oncology research is to identify aberrant gene hypermethylation associated with carcinogenesis. These findings may lead to new methods of diagnosis and treatment based on control of methylation, including new approaches to treatment of endometrial cancer.

\section{References}

1. Baylin SB and Herman JG: DNA hypermethylation in tumorigenesis: epigenetics joins genetics. Trends Genet 16: 168-174, 2000 .
2. Esteller M, Corn PG, Baylin SB and Herman JG: A gene hypermethylation profile of human cancer. Cancer Res 61: 3225-3229, 2001

3. Goldberg AD, Allis CD and Bernstein E: Epigenetics: a landscape takes shape. Cell 128: 635-638, 2007.

4. Ushijima T: Detection and interpretation of altered methylation patterns in cancer cells. Nat Rev Cancer 5: 223-231, 2005.

5. Gal-Yam EN, Jeong S, Tanay A, Egger G, Lee AS and Jones PA: Constitutive nucleosome depletion and ordered factor assembly at the GRP78 promoter revealed by single molecule footprinting. PLoS Genet 2: 160, 2006.

6. Appanah R, Dickerson DR, Goyal P, Groudine M and Lorincz MC: An unmethylated 3' promoter-proximal region is required for efficient transcription initiation. PLoS Genet 3: 27, 2007.

7. Jones PA and Baylin SB: The epigenomics of cancer. Cell 128: 683-692, 2007.

8. Toyota M, Ahuja N, Ohe-Toyota M, Herman JG, Baylin SB and Issa JP: CpG island methylator phenotype in colorectal cancer. Proc Natl Acad Sci USA 96: 8681-8686, 1999.

9. Kanaya T, Kyo S, Maida Y, Yatabe N, Tanaka M, Nakamura M and Inoue M: Frequent hypermethylation of MLH1 promoter in normal endometrium of patients with endometrial cancers. Oncogene 22: 2352-2360, 2003.

10. Banno K, Yanokura M, Susumu N, Kawaguchi M, Hirao N, Hirasawa A, Tsukazaki K and Aoki D: Relationship of aberrant DNA hypermethylation of cancer-related genes with carcinogenesis of endometrial cancer. Oncol Rep 16: 1189-1196, 2006.

11. Yang HJ, Liu VW, Wang Y, Tsang PC and Ngan HY: Differential DNA methylation profiles in gynecological cancers and correlation with clinico-pathological data. BMC Cancer 6: 212, 2006.

12. Sakaguchi J, Kyo S, Kanaya T, Maida Y, Hashimoto M, Nakamura M, Yamada K and Inoue M: Aberrant expression and mutations of TGF-beta receptor type II gene in endometrial cancer. Gyneol Oncol 98: 427-433, 2005.

13. Whitcomb BP, Mutch DG, Herzog TJ, Rader JS, Gibb RK and Goodfellow PJ: Frequent HOXA1 1 and THBS 2 promoter methylation, and a methylator phenotype in endometrial adenocarcinoma. Clin Cancer Res 9: 2277-2287, 2003.

14. Sasaki M, Kaneuchi M, Sakuragi N and Dahiya R: Multiple promoters of catechol-O-methyltransferase gene are selectively inactivated by $\mathrm{CpG}$ hypermethylation in endometrial cancer. Cancer Res 63: 3101-3106, 2003.

15. Fiegl H, Gattringer C, Widschwendter A, Schneitter A, Ramoni A, Sarlay D, Gaugg I, Goebel G, Müller HM, Mueller-Holzner E, Marth C and Widschwendter M: Methylated DNA collected by tampons - a new tool to detect endometrial cancer. Cancer Epidemiol Biomarkers Prev 13: 882-888, 2004.

16. Toyota M, Sasaki Y, Satoh A, Ogi K, Kikuchi T, Suzuki H, Mita H, Tanaka N, Itoh F, Issa JP, Jair KW, Schuebel KE, Imai K and Tokino T: Epigenetic inactivation of CHFR in human tumors. Proc Natl Acad Sci USA 100: 7818-7823, 2003.

17. Esteller M and Herman JG: Generating mutations but providing chemosensitivity: the role of O6-methylguanine DNA methyltransferase in human cancer. Oncogene 23: 1-8, 2004.

18. Kim JS, Kim JW, Han J, Shim YM, Park J and Kim DH: Cohypermethylation of p16 and FHIT promoters as a prognostic factor of recurrence in surgically resected stage I non-small cell lung cancer. Cancer Res 66: 4049-4054, 2006.

19. Mori T, Martinez SR, O'Day SJ, Morton DL, Umetani N, Kitago M, Tanemura A, Nguyen SL, Tran AN, Wang HJ and Hoon DS: Estrogen receptor-alpha methylation predicts melanoma progression. Cancer Res 66: 6692-6698, 2006.

20. Abe M, Ohira M, Kaneda A, Yagi Y, Yamamoto S, Kitano Y, Takato T, Nakagawara A and Ushijima T: CpG island methylator phenotype is a strong determinant of poor prognosis in neuroblastomas. Cancer Res 65: 828-834, 2005.

21. Waki T, Tamura G, Sato M and Motoyama T: Age-related methylation of tumor suppressor and tumor-related genes: an analysis of autopsy samples. Oncogene 22: 4128-4133, 2003.

22. Maekita T, Nakazawa K, Mihara M, Nakajima T, Yanaoka K, Iguchi M, Arii K, Kaneda A, Tsukamoto T, Tatematsu M, Tamura G, Saito D, Sugimura T, Ichinose M and Ushijima T: High levels of aberrant DNA methylation in Helicobacter pylori-infected gastric mucosae and its possible association with gastric cancer risk. Clin Cancer Res 12: 989-995, 2006. 
23. Ushijima T: Epigenetic field for cancerization. J Biochem Mol Biol 40: 142-150, 2007.

24. Daskalakis M, Nguyen TT, Nguyen C, Guldberg P, Köhler G, Wijermans P, Jones PA and Lübbert M: Demethylation of a hypermethylated P15/INK4B gene in patients with myelodysplastic syndrome by 5 -Aza-2'-deoxycytidine (decitabine) treatment. Blood 100: 2957-2964, 2002 .

25. Issa JP, Garcia-Manero G, Giles FJ, Mannari R, Thomas D, Faderl S, Bayar E, Lyons J, Rosenfeld CS, Cortes J and Kantarjian HM: Phase 1 study of low-dose prolonged exposure schedules of the hypomethylating agent 5-aza-2'-deoxycytidine (decitabine) in hematopoietic malignancies. Blood 103: 1635-1640, 2003.

26. Esteller M, Garcia-Foncillas J, Andion E, Goodman SN, Hidalgo OF, Vanaclocha V, Baylin SB and Herman JG: Inactivation of the DNA-repair gene MGMT and the clinical response of gliomas to alkylating agents. N Engl J Med 343: 1350-1354, 2000.

27. Satoh A, Toyota M, Itoh F, Sasaki Y, Suzuki H, Ogi K, Kikuchi T, Mita H, Yamashita T, Kojima T, Kusano M, Fujita M, Hosokawa M, Endo T, Tokino T and Imai K: Epigenetic inactivation of CHFR and sensitivity to microtubule inhibitors in gastric cancer. Cancer Res 63: 8606-8613, 2003.

28. Scolnick DM and Halazonetis TD: CHFR defines a mitotic stress checkpoint that delays entry into metaphase. Nature 406: 430-435, 2000
29. Kang D, Chen J, Wong J and Fang G: The checkpoint protein CHFR is a ligase that ubiquitinates Plk1 and inhibits Cdc2 at the G2 to M transition. J Cell Biol 156: 249-259, 2002.

30. Yu X, Minter-Dykhouse K, Malureanu L, Zhao WM, Zhang D, Merkle CJ, Ward IM, Saya H, Fang G, van Deursen J and Chen J: CHFR is required for tumor suppression and Aurora A regulation. Nat Genet 37: 401-406, 2005.

31. Ogi K, Toyota M, Mita H, Satoh A, Kashima L, Sasaki Y, Suzuki H, Akino K, Nishikawa N, Noguchi M, Shinomura Y, Imai $\mathrm{K}$, Hiratsuka $\mathrm{H}$ and Tokino T: Small interfering RNAinduced CHFR silencing sensitizes oral squamous cell cancer cells to microtubule inhibitors. Cancer Biol Ther 4: 773-780, 2005.

32. Banno K, Yanokura M, Kawaguchi M, Kuwabara Y, Akiyoshi J, Kobayashi Y, Iwata T, Hirasawa A, Fujii T, Susumu N, Tsukazaki K and Aoki D: Epigenetic inactivation of the CHFR gene in cervical cancer contributes to sensitivity to taxanes. Int J Oncol 31: 713-720, 2007.

33. Koga Y, Kitajima Y, Miyoshi A, Sato K, Sato S and Miyazaki K: The significance of aberrant CHFR methylation for clinical response to microtubule inhibitors in gastric cancer. J Gastroenterol 41: 133-139, 2006. 